\title{
On the use of artificial excitation in operational transfer path analysis
}

\author{
N.B. Roozen ${ }^{\mathrm{a}, \mathrm{b}}$, Q. Leclère ${ }^{\mathrm{c}}$ \\ ${ }^{a}$ Delft University of Technology, Faculty of Aerospace Engineering, Kluyverweg 1, 2629 \\ HS Delft, Netherlands \\ ${ }^{b}$ Katholieke Universiteit Leuven, Department of Mechanical Engineering, Box 2420, \\ Celestijnenlaan 300 B, B-3001 Leuven, Belgium \\ ${ }^{c}$ Laboratoire Vibrations Acoustique, INSA Lyon, 25 bis avenue Jean Capelle \\ F-69621 Villeurbanne Cedex, FRANCE
}

\begin{abstract}
A new measurement procedure to obtain transmissibilities for application in a two-step transfer path analysis is presented. In the proposed method an external excitation, for instance by means of a non-instrumented hammer, is used to bring the structure into vibration. The resulting response is then used to estimate the transmissibilities. In most cases the transmissibilities thus determined are of a better quality as compared to the conventionally determined transmissibilities obtained during machine operation, i.e. in conventional operational transfer path analysis procedures. The reason of this is that when a structure is excited by hammer strokes, its responses are largely independent from each other, whilst vibrations induced by machine operation are in general not. The hammer stroke measurements can be performed relatively easy whilst the experimental effort is relatively low.

Subsequently the transmissibilities are applied in a transfer path analysis (TPA) like approach. It was found that the identification of the transfer paths were better as compared to the identification transfer paths by means of a conventional operational transfer path analysis. The advantages of the method are illustrated by means of an experiment on a small gearbox.

Additionally, a procedure to determine the number of significant transmission paths is proposed which is based upon a singular value decomposition
\end{abstract} Roozen)

Email address: n.b.roozen@tudelft.nl; bert.roozen@mech.kuleuven.be (N.B. 
of the response matrix. In the application discussed in the paper the number of significant structural transmission paths could be estimated by means of this procedure, as well as the order of magnitude of the strength of the acoustic path relative to that of the structural transmission paths.

Keywords: transfer path analysis, operational, transmissibilities

\section{Introduction}

Transfer path analysis is of general interest to noise control engineers. In literature a large number of methods are described, all having their specific advantages and disadvantages.

Transfer path analysis methods can be divided into two groups: one-step methods and the two-step methods. The former estimates the significance of the individual transfer paths from a single set of measurements, with the machine in operation, often running at different operational speeds and loads to obtain sufficient information. Examples of one-step methods are operational transfer path analysis techniques (OTPA) [1, 2], and the force analysis technique (FAT) [3]. In the two-step methods, relations between exciting forces and responses (e.g. transfer functions), or relations between responses and responses (e.g. transmissibility functions), are estimated in a first step, most often with the machine not in operation, sometimes even dismounted. In a second step operational measurements are conducted for which situation the individual transmission path strengths are to be estimated. Examples of two-step methods are indirect force identification methods, e.g. the mount stiffness method [4], the pseudo force methodology [5, 6, 7], force transmission path analysis techniques (TPA) [4] (see for a nice overview [8]), and yet another variant of TPA, the so-called Global Transfer Direct Transfer (GTDT) method $[9,10,11]$. For instance, in the pseudo force methodology [5, 6], excitation forces are estimated based on transfer function measurements (step 1) in combination with operational response measurements (step 2).

A method that could be classified as a hybrid one-step / two-step approach is OPAX [12], which combines transfer path measurements with operational measurements. In the OPAX method mounts connecting the source and receiver are used as force sensors, which are calibrated using parametric laws during a first step using artificial excitations. The OPAX approach can be considered as an enhancement of TPA that does not require to uncouple the source and receiver. 
Lately the one-step methods, such as operational transfer path analysis techniques (OTPA), have gained a renewed interest in the engineering community. The reason for this renewed interest is obvious; in comparison with the two-step methods, OTPA does not require the vibration source to be removed, which is usually a huge effort and also modifies the dynamic behavior of the passive part. Instead, one-step methods such as OTPA identify the main transmission paths through an estimation of the transmissibilities under 'operational' conditions, i.e. when the machine is in operation. There is no need to remove the vibration source, which saves a lot of time and money.

Of course, one-step methods like OTPA suffer from technical drawbacks as compared to two-step methods such as TPA. The main issue is that most one-step methods, such as OTPA, use matrix transmissibilities as introduced by Ribeiro [13]. Transmissibilities are inherently different from transfer functions, e.g. mobility transfer functions. Knowing the transfer functions from the exciting forces to the response points, as being estimated in two-step methods such as TPA, the exciting forces of the sources can be identified. The response can be written as a linear sum of individual source contributions, i.e. there exists a causal relationship. When dealing with transmissibilities, however, the response can not be written as a linear sum of individual 'vibration' contributions, due to the inherent coupling between input measurement points. This means that the transmissibility matrix concept should be used with care to identify the noise and vibration sources [1]. However, from an engineering point of view, the transmissibility matrix concept can still be very useful to identify significant sources of vibration at particular frequencies. In literature many examples can be found in which the transmissibility matrix concept is applied successfully (see e.g. $[2,14,15]$ ).

In this paper a two-step transmissibility-based measurement procedure is proposed in which it is not required to remove the vibration source. To this respect, the method is similar to OTPA, albeit that it is not a one-step method. One of the difficulties in OTPA is that it can be difficult to obtain a data set that is sufficiently linearly independent to enable a successful identification of the transmissibility matrix. In practice, it means that operating conditions have to be sufficiently distinctive to generate different operational deflection shapes, at each frequency bin, requiring a rather large amount of measurements. In the proposed two-step transmissibility-based measurement procedure the transmissibility matrix is solely computed from a set of vibroacoustic responses resulting from an artificial excitation only, e.g. by means of a hammer, spanner or screwdriver, with the machine not in operation. 
Such an experiment is easy to do, with relatively less efforts as compared to conventional two-step TPA approach, as there is no need to disassemble the structure. Compared to conventional OTPA it is believed that the proposed method is also advantageous as it will require less data sets to obtain a full rank, invertible system, thus also reducing the experimental effort.

\section{Transmissibility matrix estimation; an alternative approach}

Let's consider a vibro-acoustic system, consisting of two parts, i.e. an active part that causes the vibro-acoustic excitation and a passive part, as illustrated in Fig. 1. A number of transmission paths can be responsible for the structure-borne transmission, for instance a mechanical connection between the two parts of the vibro-acoustic system. Acoustic transmission paths can be considered equally well, but will be omitted in this paper for simplicity. The forces acting between the two system parts due to the mechanical transmission paths are denoted by a vector $\mathbf{f}$.

Within the passive part of the system a number of vibro-acoustic responses are chosen, usually close to the interface between the active and passive parts, as being representative for the vibro-acoustic transfer paths. Let these indicator responses be denoted by $\mathbf{y}$. Furthermore, another set of vibro-acoustic responses, referred to as the target responses $\mathbf{x}$, are defined on the passive system, usually at a larger distance from the interface between the active and passive parts. Note that the variables $\mathbf{x}, \mathbf{y}$ and $\mathbf{f}$ are defined in the frequency domain, and their dependency on frequency is omitted for brevity.

Defining the vibro-acoustic response $\mathbf{x}$ and $\mathbf{y}$ of the system in terms of the interface force $\mathbf{f}$ as follows

$$
\begin{aligned}
& x=\mathbf{H f} \\
& y=\boldsymbol{\Phi f}
\end{aligned}
$$

where $\mathbf{H}$ and $\boldsymbol{\Phi}$ are transfer functions of the system, and defining the transmissibility matrix $\mathbf{T}$ as the matrix relation between the vibro-acoustic response $\mathbf{x}$ and $\mathbf{y}$ as

$$
\mathrm{x}=\mathrm{Ty}=\mathbf{T} \Phi \mathbf{f}
$$

we can write [16]: 


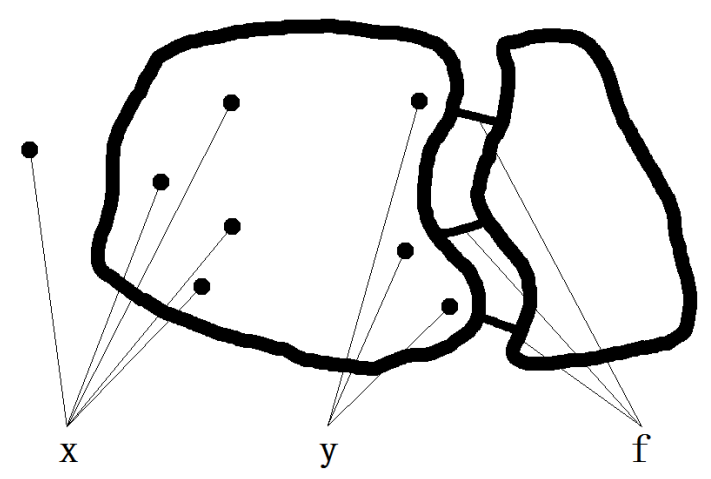

Figure 1: Passive and active vibro-acoustic sub systems. Left: passive part, right: active part.

$$
\mathbf{T}=\mathbf{H} \boldsymbol{\Phi}^{-\mathbf{1}}
$$

The transmissibility matrix $\mathbf{T}$ uniquely exists only if the matrix $\mathbf{\Phi}$ is square and invertible, which is the case when the number of references equals the number of forces.

Eq. 4 is a formal way to compute the transmissibility matrix $\mathbf{T}$, as it requires the measurement of the transfer functions $\mathbf{H}$ and $\boldsymbol{\Phi}$. In OTPA the transmissibility matrix $\mathbf{T}$ is computed from a set of operational vibro-acoustic responses. These sets of measurement data are contained in the matrices $\mathbf{X}$ and $\mathbf{Y}$, each column of these matrices containing the response of the system, $\mathrm{M}$ in total.

$$
\begin{aligned}
& \mathbf{X}=\left[\mathbf{x}^{(\mathbf{1})} \mathbf{x}^{(2)} \ldots \mathbf{x}^{(\mathbf{M})}\right] \\
& \mathbf{Y}=\left[\mathbf{y}^{(\mathbf{1})} \mathbf{y}^{(\mathbf{2})} \ldots \mathbf{y}^{(\mathbf{M})}\right]
\end{aligned}
$$
by

Having obtained these data, the transmissibility matrix $\mathbf{T}$ is calculated

$$
\mathbf{T}=\mathbf{X ~ Y ^ { \prime }}\left(\mathbf{Y ~ Y}^{\prime}\right)^{-1}=\mathbf{X ~ Y ^ { + }}
$$


where ' denotes the conjugate transpose operation and where ${ }^{+}$denotes the pseudo-inverse. Note that for the pseudo-inverse $\mathbf{Y}^{+}$to be computed correctly, the number of columns of $\mathbf{Y}, M$ (i.e. the number of artificial excitation experiments), needs to be larger than or equal to the number of rows (i.e. the number of measurement signals contained in $\mathbf{y}$ ). Moreover, the different columns have to be sufficiently linearly independent to ensure matrix $\mathbf{Y} \mathbf{Y}^{\prime}$ to be of full rank.

Following $[1,15,20]$ the transmissibilities can also be estimated from auto and cross-spectra estimates. Writing Eq. 3 as $\mathbf{X}=\mathbf{T} \mathbf{Y}$ and post multiplying

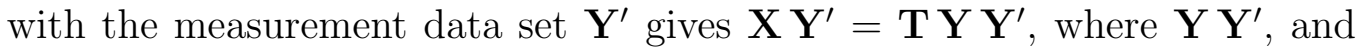

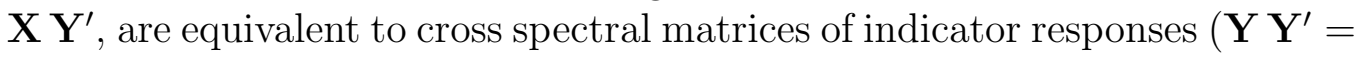

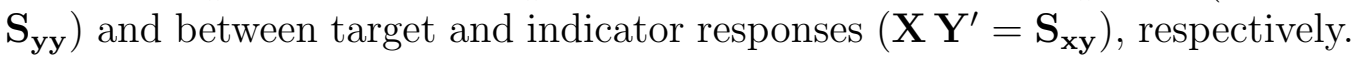
This leads to a $H_{1}$-like expression of $\mathbf{T}$, which is equivalent to Eq. 7 :

$$
\mathrm{T}=\mathrm{S}_{\mathrm{xy}} \mathrm{S}_{\mathbf{y y}}^{-1}
$$

Obviously, the $\mathbf{S}_{\mathbf{y y}}$ matrix has to be invertible. To make the $\mathbf{S}_{\mathbf{y y}}$ matrix invertible, averaging of measurements over varying operating conditions is practically speaking always required.

Likewise, an $H_{2}$ or, even better, an $H_{s}$ estimate could be used for the estimation of the transmissibility matrix, as suggested in [21], arguing that for operational responses the signal-to-noise ratio on $\mathbf{X}$ and $\mathbf{Y}$ are likely to be of the same order of magnitude (provided that the same type of sensors are used).

The alternative approach suggested in this paper is not to use operational vibro-acoustic responses to estimate the transmissibility matrix, like is done in the conventional OTPA procedure, but to excite the structure externally at a number of suitable locations whilst the machine is not in operation. The external excitation can for instance be done by means of a hammer. This hammer is not required to be instrumented with a force transducer for this purpose. The transmissibility matrix can be estimated by means of Eq. 7, or more specifically by means of Eq. 8 .

To distinguish between the proposed estimate and the OTPA estimate, the hammer excited forced vibration estimate will be denoted by $\mathbf{T}_{\mathbf{h}}$ and the OTPA estimate of the transmissibility matrix will be denoted by $\mathbf{T}_{\mathbf{o}}$. Furthermore, a reference transmissibility matrix $\mathbf{T}_{\text {ref }}$ is defined, which is based on Eq. 4. This method requires a TPA-like approach to measure the transfer functions $\mathbf{H}$ and $\boldsymbol{\Phi}$, and is for that reason normally not available. However, 
as in the experimental test set-up used for this paper force transducers were incorporated, an exact transmissibility matrix can be determined as a reference.

It will be shown in section 4 that the proposed measurement procedure with hammer excitation yields better estimates of the transmissibilities as compared to the OTPA method. However, before considering the experimental application in section 4 , a method to assess the number of significant transmission paths will be discussed in the next section.

\section{Singular value decomposition as a means to identify the number of significant transmission paths}

When identifying transfer paths by means of a transmissibility matrix method, all sources should be accounted for by means of indicator responses [22]. To this respect it should be remembered that matrix transmissibilities are not system characteristics but depend on the position and number of excitations acting on the system [13], underlining the importance of this requirement. Defining an incorrect number of input quantities will give an incorrect estimate of the $\mathbf{T}$ for the problem at hand.

A correctly defined set of indicator responses $\mathbf{y}$ is a vector with a dimension which is equal to the number of excitations contained in $\mathbf{f}$ [16]. Defining too many indicator responses will make the matrix $\mathbf{Y} \mathbf{Y}^{\prime}$ rank-deficient, and thus non-inversible, whilst defining too few indicator responses will make the matrix $\boldsymbol{\Phi}$ as defined in Eq. 2 and used in Eq. 4 non-inversible. In the first case an estimation of the transmissibility matrix by means of Eq. 7 is impossible, whilst in the second case the transmissibility matrix $\mathbf{T}$ does not exists. So in order to be able to quantify the relevant transmission paths it is essential to use the correct number of indicator responses.

To check the correctness of the chosen number indicator responses $\mathbf{y}$, it is very useful to compute a singular value decomposition of the matrix $\left[\mathbf{X}^{\prime} \mathbf{Y}^{\prime}\right]^{\prime}$ as defined in Eq. 5 and 6 , containing the $M$ data sets. Indeed, denoting $\left[\mathbf{X}^{\prime} \mathbf{Y}^{\prime}\right]^{\prime}$ by $\mathbf{Z}$, we can write

$$
\mathrm{Z}=\left[\begin{array}{l}
\mathbf{X} \\
\mathbf{Y}
\end{array}\right]=\left[\begin{array}{l}
\mathbf{H} \\
\mathbf{\Phi}
\end{array}\right] \mathbf{F}
$$

It can be seen that all columns of the matrix $\mathbf{Z}$ are linear combinations of the columns of matrix $\left[\mathbf{H}^{\prime} \boldsymbol{\Phi}^{\prime}\right]^{\prime}$. Thus, the rank of $\mathbf{Z}$ is lower or equal to the number of excitations acting on the mechanical system. If correctly defined, 
the rank of $\mathbf{Z}$ is equal to the number of excitations acting on the mechanical system. The inspection of this rank from operational data can also be seen as an indirect way of assessing the number of paths.

The matrices $\mathbf{H}, \boldsymbol{\Phi}$ and $\mathbf{F}$ are unknown, but if the number of assumed excitations is $n$, then the rank of $\mathbf{Z}$ cannot be greater than $n$. If the rank is greater than $n$ a path has been omitted. Obviously, in this case the number of assumed excitations $n$ is too low as compared to the actual number of physical paths. If the rank is lower than $n$ the identification of $\mathbf{T}$ is not possible.

In conclusion, performing a singular value decomposition of the response matrix $\mathbf{Z}$ with $M$ measurement data sets, allows a posterior determination of the number of transmission paths involved.

Note that the proposed method is not a virtual source analysis method, aiming at an identification of the number of incoherent sources exciting the system $[17,18,19,23]$. Instead, the proposed method is based on several (artificial) excitation configurations, and aims at the identification of the number of transmission paths. The former is a blind analysis of the inputs, the latter a blind analysis of the transfer system.

The application of this concept, as well as the alternative measurement procedure to estimate the transmissibility matrix, as presented in Section 2, will be demonstrated for a practical test set-up in the next section.

\section{Experimental application on a gear-box}

In this section the proposed measurement procedure to obtain transmissibilities, $T_{h}$, as defined in Secion 2 is applied experimentally and its predictive performance is compared to $T_{r e f}$ and $T_{o}$ to illustrate its effectiveness. In the proposed method the structure is brought into vibration by means of a hammer excitation without the need to measure the exciting forces. The resulting response is then used to determine the transmissibilities. Furthermore the proposed procedure to determine the number of significant transmission paths as presented in Section 3 will be applied in an experiment.

The organization of this section is as follows. The test set-up is discussed in section 4.1. The number and location of the indicator responses $\mathbf{y}$ which are appropriate for the estimation of the transfer paths is discussed in section 4.2 using the proposed procedure to estimate the number of significant transmission paths. In section 4.3 a reference transmissibility matrix is determined by means of the force transducers that are incorporated in 
the test set-up. In section 4.4 transmissibility matrices are estimated in a conventional way from operational machine vibrations and from the hammer excitation vibrations. In this section a comparison with the reference transmissibility matrix is given as well. In section 4.5 the application of the thus determined transmissibility matrices in an operational transfer path analysis (OTPA) is given.

\subsection{Test set-up}
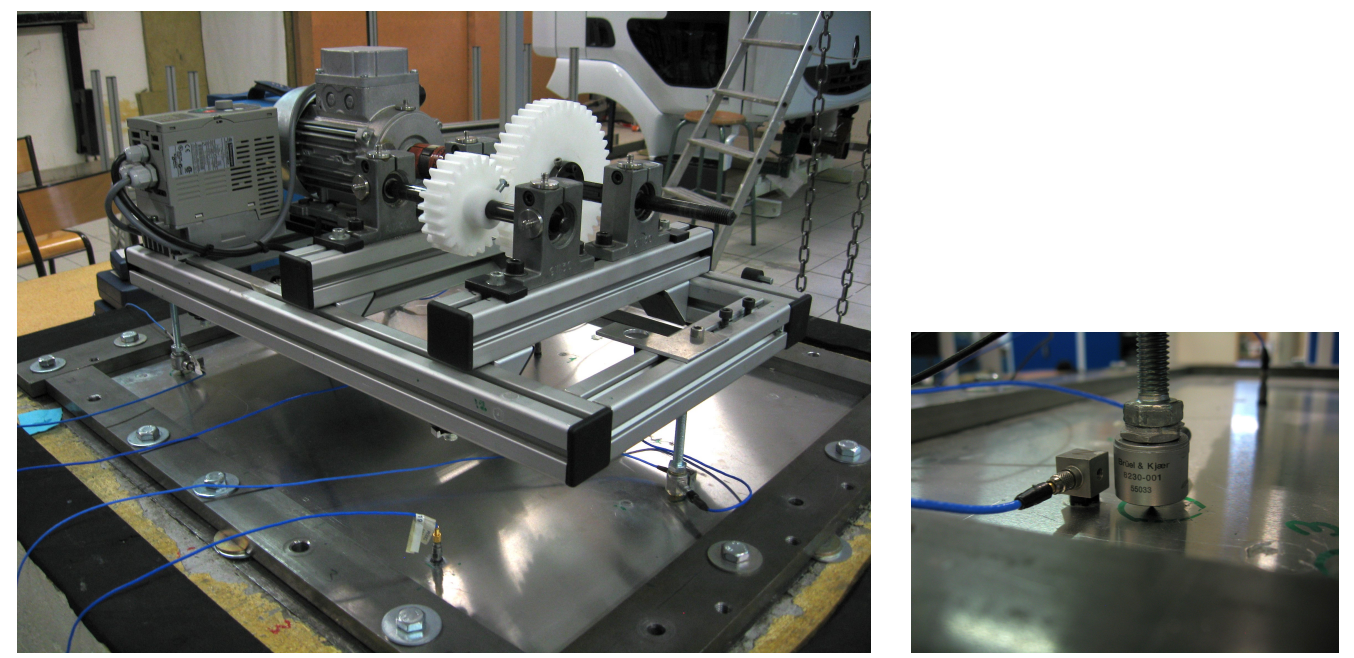

(a) Small gearbox mounted on a frame, frame standing on (b) Detail showing the force a plate by means of three pins transducer integrated in the pin and a $3 \mathrm{D}$ accelerometer

Figure 2: Test set-up.

The test set-up comprises a frame structure holding a set of gears driven by an electro-motor, as shown in Figure 2(a). Figure 2(b) shows a detail of the rigid pins, three in total, with which the frame structure is resting on a flexible aluminum plate with a thickness of approximately $3 \mathrm{~mm}$. The plate is damped by means of a piece of felt lying on the plate (note, however, that the felt is removed when taking pictures of the set-up). In each pin, a force transducer is mounted, which allows the transmitted force in the direction normal to the plate to be measured. The data obtained with the force transducers will be used for verification purposes only. 3D-Accelerometers are mounted on the plate close to the three pins to measure the indicator responses $\mathbf{y}$ in the three translation directions, i.e. normal to the plate and in the two in-plane 
directions. In addition another three 1D-accelerometers are mounted on the plate to measure the vibrations of the plate in normal direction, being the target responses $\mathbf{x}$. One of the accelerometers to measure the target response can be seen in Figure 2(a).

\subsection{Identification of the number of transfer paths by means of singular value decomposition}

The first and most crucial choice that needs to be made is the selection of the number and the position of the indicator responses $\mathbf{y}$ which are appropriate for the estimation of the transfer paths. It was decided to use accelerometer transducers only, no acoustic transducers. The validity of this choice, and the number and position of the accelerometers, can be checked by means of a singular value decomposition of the response matrix $\mathbf{Z}$ (defined by Eq. 9), as discussed in Section 3. Two types of response matrices $\mathbf{Z}$ were obtained, i.e. by means of a conventional operational measurement and by means of a hammer excitation. The matrices will be denoted by $\mathbf{Z}_{\mathbf{o}}$ and $\mathbf{Z}_{\mathbf{h}}$, respectively.

In the test set-up three 3D-accelerometers are used on the plate, near the rigid pins, yielding 9 reference signals. As target responses the accelerations in normal direction of the plate at 3 arbitrary positions were taken. Collecting all the operational data of 15 different motor speeds in a response matrix $\mathbf{Z}_{\mathbf{o}}$, keeping all PCA-eigenvectors $(\mathrm{PCA}=$ principal component analysis, see for instance [23]) extracted from each operating point, and 15 different responses to hammer excitation in a response matrix $\mathbf{Z}_{\mathbf{h}}$, a singular value decomposition of both matrices was determined.

To determine the significance of the acoustic path, which was not accounted for in this set-up, yet another experiment was conducted in which the machine was lifted about $1 \mathrm{~cm}$. In this situation the machine is not in mechanical contact with the plate, which leaves the acoustic path as the only possible way to excite the plate structure. The singular values corresponding to the measurement data sets thus obtained during operation of the machine, using the same indicator responses and target vibro-acoustic responses of the plate, can be used to assess the magnitude of the acoustic path in relation to the mechanical transmission paths. The most significant singular values of this configuration are denoted by $\mathbf{s v}_{\text {lifted }}$.

Figure 3 and Figure 4 show the normalized singular values of the matrices $\mathbf{Z}_{\mathbf{o}}$ and $\mathbf{Z}_{\mathbf{h}}$, respectively. Figure 3 shows $\mathbf{s v}_{\text {lifted }}$ as well (thick black line). Normalization is done relative to the sum of the singular values at each 


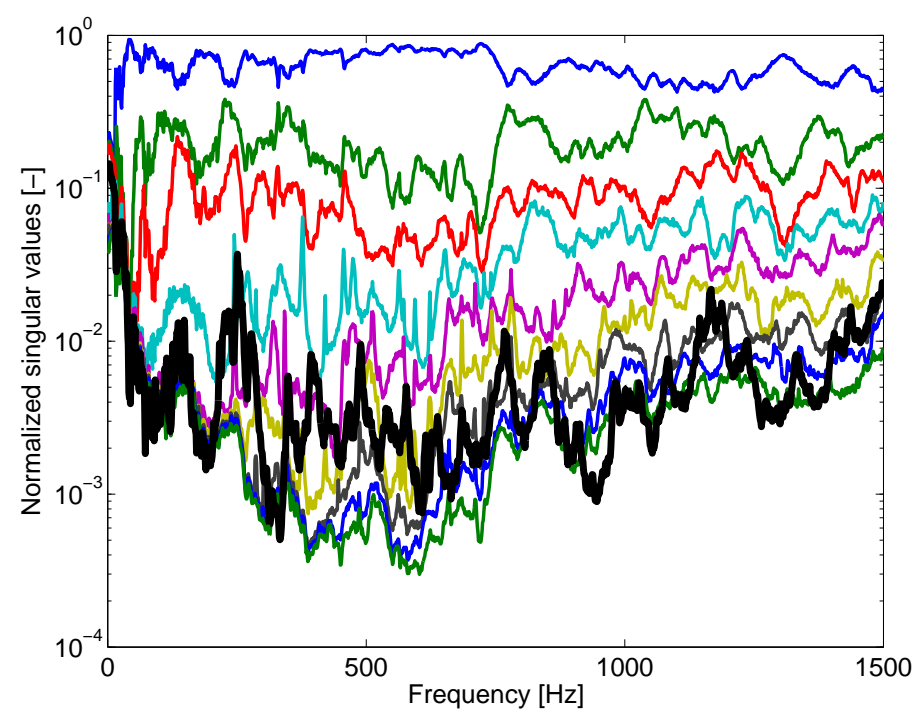

Figure 3: The first 9 normalized singular values of matrix $\mathbf{Z}_{\mathbf{o}}$ (without PCA denoising) and $\mathbf{s v}_{\text {lifted }}$ (thick black line).

frequency bin. As we have 9 indicator responses (XYZ responses at the three pins) and 3 target responses, 12 singular values were computed at each frequency. In Figure 3 and Figure 4 only the first 9 singular values are shown for clarity.

From Figure 3 it can be seen that three to six singular values are above $\mathbf{s v}_{\text {lifted }}$, depending upon frequency. These singular values must all correspond to structural transmission paths because $\mathbf{S v}_{\text {lifted }}$ corresponds to the most significant acoustic transmission path. Comparing Figure 3 with Figure 4 it can be seen that the ratio between the third and the fourth singular values is larger for $\mathbf{Z}_{\mathbf{h}}$ than for $\mathbf{Z}_{\mathbf{o}}$, meaning that during hammer excitation is was more easy to generate linearly independent excitations for the three most dominant transmission paths, exciting less the other non-dominant paths. Using engineering judgement, the first three dominant transmission paths are likely to correspond to the vibration paths of the three pins in normal direction to the plate. The fourth and higher singular values are likely to correspond to tangential paths, i.e. transmission paths in which the tangential motion of the plate is involved. These paths are particularly affected by the bending modes of rods, causing peaks in the singular values (e.g. at 380 $\mathrm{Hz})$.

In conclusion, in the low frequency range up to approximately $650 \mathrm{~Hz}$ 


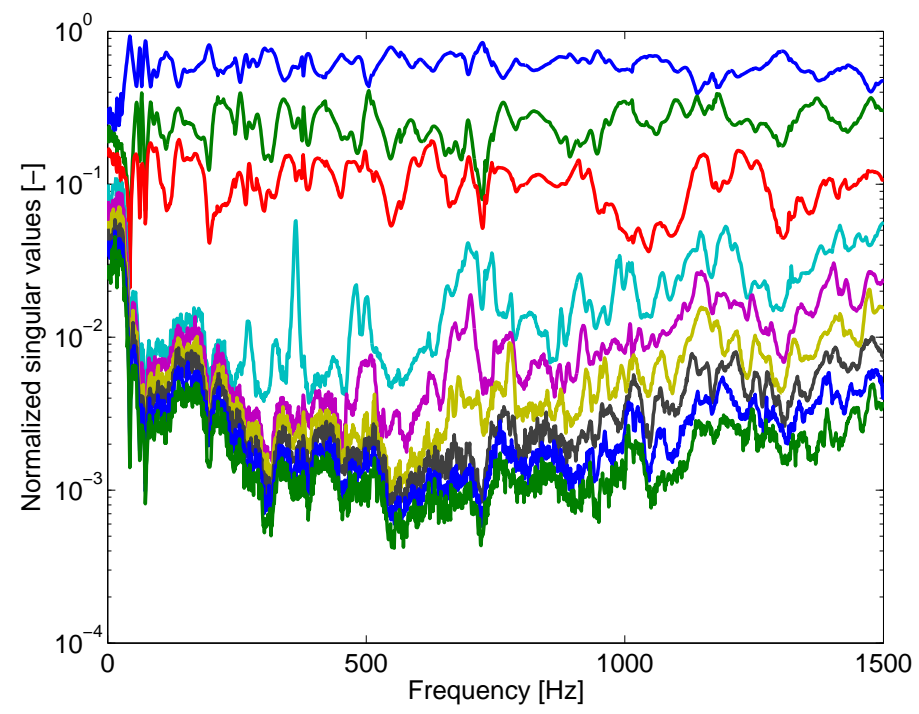

Figure 4: The first 9 normalized singular values of matrix $\mathbf{Z}_{\mathbf{h}}$.

three structural transmission paths are most dominant. At this frequency and below this frequency the ratio between the third and the fourth singular values is more than a factor 6 (for $\mathbf{Z}_{\mathbf{h}}$ ), expect for a few specific frequencies like $380 \mathrm{~Hz}$. These three transmission paths are likely to correspond to vibrations at the connection points in normal direction of the plate. At higher frequencies more transmission paths seem to be involved, although the three mentioned paths remain the most important transmission paths, which can especially be seen from Figure 4. Because of this, three accelerometers near the connection points that measure the vibration of the plate in normal direction is sufficient for this application.

Furthermore, it can be stated that a unique, and also a practically useful transmissibility matrix can be defined for this case between the three vibrations of the pin in the direction normal to the plate and some arbitrarily defined points on the passive part of the system, as the number of references equals the number of forces (ref. Eq. 4).

A PCA is performed [23] to retain the first three principal components only, using only the accelerations near the three pins in normal direction as the indicator responses. This is justified as in each separate measurement data set the number of principal components we are interested in from a physical point of view can never exceed three and because we are interested 
in the transmission paths in normal direction only. The PCA procedure effectively suppresses the other structural and acoustic transmission paths as well as the noise components as good as possible. The resulting response matrix $\mathbf{Z}$ consists of $15^{*} 3=45$ columns ( 15 operating conditions, 3 principal components). Its singular values are shown in Figure 5. It can be seen that, as compared to Figure 3 the first three singular values are not noticeably affected, whilst the higher singular values (SV4 and above) are significantly reduced.

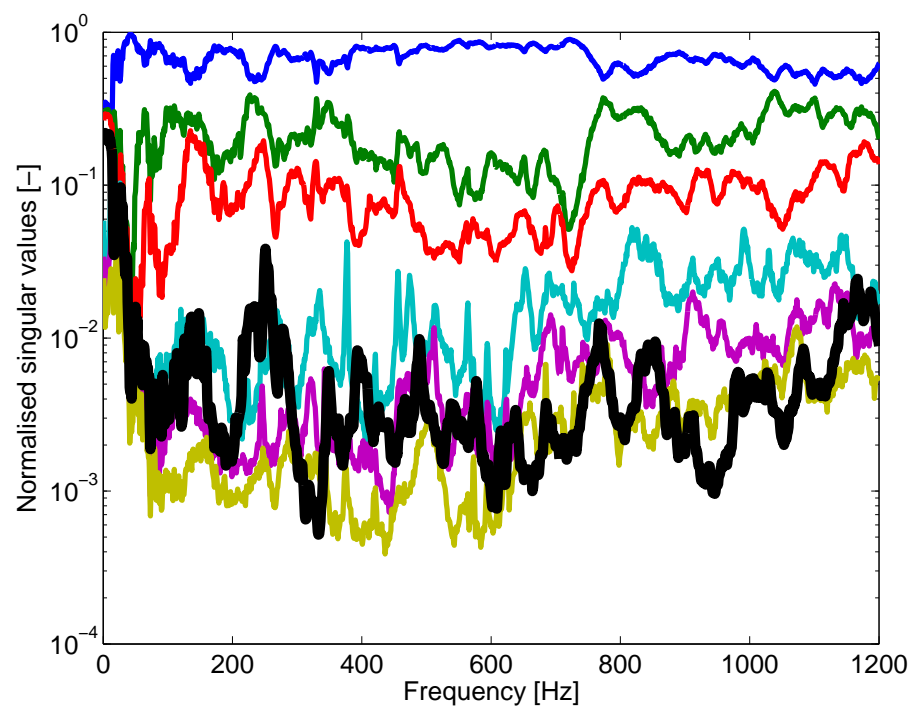

Figure 5: Normalized singular values of matrix $\mathbf{Z}_{\mathbf{o}}$ (with PCA denoising, using 3 input channels in normal direction, retaining 3 PC's) and $\mathbf{s v}_{\text {lifted }}$ (thick black line).

\subsection{Estimation of the reference transmissibility matrix}

It was concluded in the previous section that a unique, and also a practically useful transmissibility matrix can be defined between the three vibrations of the pin in the direction normal to the plate and some arbitrarily defined points on the passive part of the system, as the number of references equals the number of forces (ref. Eq. 4). To compare the measured transmissibilities $b f T_{o}$ and $b f T_{h}$ a reference transmissibility matrix $b f T_{r e f}$ will be determined. The reference transmissibilities $\mathbf{T}_{\text {ref }}$ will be determined by means of Eq. 4, thus requiring the knowledge of the transfer matrices $\mathbf{H}$ and $\boldsymbol{\Phi}$. 
The transfer functions $\mathbf{H}$ and $\boldsymbol{\Phi}$ are determined by means of the force transducers mounted in the three pins. Whilst hitting the active part of the structure (the frame structure, as shown in Figure 2(a)) with a noninstrumented hammer at $M=15$ several positions in vertical and horizontal directions, both the acceleration response of the plate structure and the forces in the three pins were measured simultaneously, taking the phase of one accelerometer as a reference. The accelerations at different operational conditions (i.e. the 15 hits with the hammer) at the target points are collected in a matrix $\mathbf{X}$ consisting of columns with acceleration data. In a similar manner the accelerations at different operational conditions at the indicator points are collected in a matrix $\mathbf{Y}$, and the forces measured in the three pins in a matrix $\mathbf{F}$. The matrices $\mathbf{X}, \mathbf{Y}$ and $\mathbf{F}$ all consists of $M$ columns with data. In a similar manner as in Eq. 7, and referring to definitions Eq. 1 and Eq. 2, we can now write:

$$
\mathbf{H}=\mathbf{X F}^{\prime}\left(\mathbf{F F}^{\prime}\right)^{-1}=\mathbf{X F}^{+}
$$

and

$$
\Phi=\mathbf{Y F}^{\prime}\left(\mathbf{F F}^{\prime}\right)^{-1}=\mathbf{Y F}^{+}
$$

The expressions in Eq. 10 and Eq. 11 give matrix $H_{1}$ estimates. With the transfer function matrices thus estimated, the reference transmissibilities $\mathbf{T}_{\text {ref }}$ can be determined, using Eq. 4 in combination with the transfer function estimates as given by Eq. 10 and Eq. 11.

It should be noted that the determination of the transferfunctions $\mathbf{H}$ and $\Phi$ as described above takes into account the effect of pre-stress of the plate structure due to the loading of the gearbox weight. Removing the gearbox and then measuring the transferfunctions by means standard measurement techniques (instrumented hammer, accelerometer) will result in erroneous transferfunctions due to mentioned effect.

\subsection{Estimation of transmissibility matrices}

With the machine standing on the plate, operational measurements were performed for 15 operational conditions, using different motor speeds. The measurement data was obtained using 39 averages, using a Hanning time window, with $50 \%$ overlap, and a spectral resolution of $1 \mathrm{~Hz}$. The transmissibility matrix $\mathbf{T}_{\mathbf{o}}$ between the indicator responses $\mathbf{y}$ and the target responses 
$\mathbf{x}$ is estimated by means of operational input data, employing Eq. 8. These measurements typically took one to two hours.

Alternatively, the frame structure, being the active part of the system, is externally excited by means of a hammer excitation at 15 different positions, obtaining 15 measurement data sets, the same number of data sets as used for $\mathbf{T}_{\mathbf{o}}$. At 7 positions the frame (see Figure 2(a)) was excited in vertical direction and at 8 positions the frame was excited in horizontal direction, equally distributed around the circumference. The electro motor was not in operation. With the vibrations thus induced the transmissibility matrix $\mathbf{T}_{\mathbf{h}}$ between $\mathbf{y}$ and $\mathbf{x}$ is estimated by means of Eq. 8 also, now using hammer forced vibration data. These measurements typically took a few minutes, which is much less demanding as compared to the operational measurements.

Estimates of the transmissibility matrix using both approaches are compared with the reference transmissibility matrix as discussed in the previous section. Figure 6 shows a typical transmissibility matrix estimate from the out-of-plane acceleration of the plate near a pin to the out-of-plane acceleration of the plate at a target position. It can be seen that, as compared to $\mathbf{T}_{\mathbf{o}}, \mathbf{T}_{\mathbf{h}}$ corresponds significantly better to $\mathbf{T}_{\text {ref }}$.

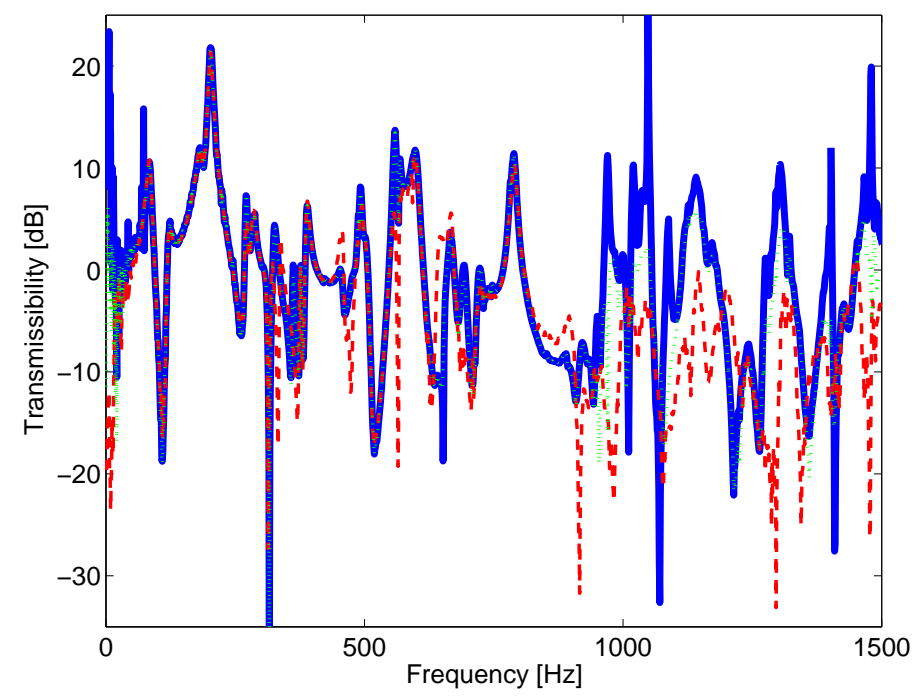

Figure 6: One element of the transmissibility matrix estimate. Solid blue line: $\mathbf{T}_{\text {ref }}$, dashed red line: $\mathbf{T}_{\mathbf{o}}$, dotted green line: $\mathbf{T}_{\mathbf{h}}$.

Figure 7 shows the global error in the estimation of the transmissibilities, which is defined as 


$$
\left\|\mathbf{T}-\mathbf{T}_{\text {ref }}\right\| /\left\|\mathbf{T}_{\text {ref }}\right\|
$$

where $\mathbf{T}$ is the transmissibility matrix estimate, either $\mathbf{T}_{\mathbf{o}}$ or $\mathbf{T}_{\mathbf{h}}$, and $\mathbf{T}_{\text {ref }}$ is the reference transmissibility matrix. By means of $\|\mathbf{x}\|$ the L2 norm of $\mathbf{x}$ is denoted. It can be seen from Figure 7 that $\mathbf{T}_{\mathbf{h}}$ estimates the reference transmissibility matrix much more better than $\mathbf{T}_{\mathbf{o}}$, the errors being approximately one order smaller, or the same, depending upon frequency.

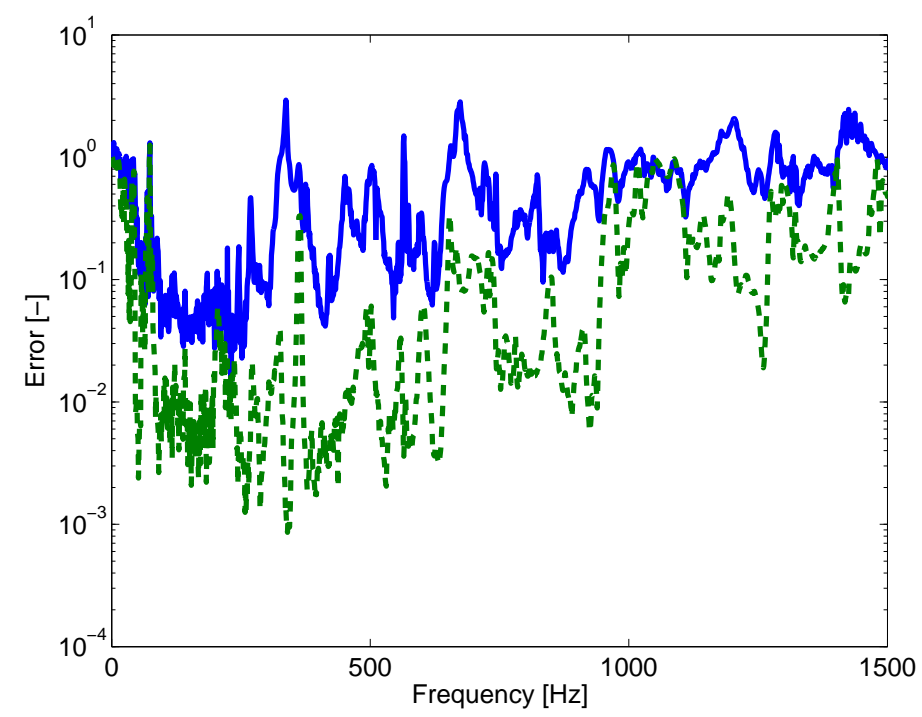

Figure 7: Transmissibility matrix errors, as defined by Eq. 12. Solid blue line: $\left\|\mathbf{T}_{\mathbf{o}}-\mathbf{T}_{\text {ref }}\right\| /\left\|\mathbf{T}_{\text {ref }}\right\|$, dashed green line: $\left\|\mathbf{T}_{\mathbf{h}}-\mathbf{T}_{\text {ref }}\right\| /\left\|\mathbf{T}_{\text {ref }}\right\|$.

The reason that the $\mathbf{T}_{\mathbf{h}}$ transmissibility matrix estimates are much better is because the structure is excited by hammer strokes which are largely independent from each other. In the conventional OTPA, yielding $\mathbf{T}_{\mathbf{o}}$, it is often difficult to obtain linearly independent operational deflection shapes even with strongly different operating conditions (i.e. at different speeds of the electro motor). This generates difficulties with the inversion of the matrix $\mathbf{Y}$ in Eq. 7, causing inaccurate estimates of the transmissibility matrix.

\subsection{Operational transfer path analysis}

Whilst the singular value decomposition of the response matrix $\mathbf{Z}$ as described in section 4.2 reveals the number of significant transfer paths, the 
ranking of the individual transfer paths is a task of a transfer path analysis method. In this section the individual contributions of the transmission paths at the three pins in normal direction to the plate are estimated quantitatively, employing the transmissibility matrix estimates as discussed in section 4.4. Estimates of the three transmission paths are obtained with conventional OTPA and hammer OTPA. Because in the measurement setup we measured the forces in normal direction at the three connection points as well, we can determine the actual contribution of each path directly, without the need of any matrix inversion. Both OTPA methods are compared with these actual contributions.

The individual contributions of the three connection points in normal direction can easily be calculated by rewriting Eq. 1 as

$$
\mathbf{x}^{(\mathbf{j})}(\mathbf{i})=\mathbf{H}(\mathbf{i}, \mathbf{j}) \mathbf{f}(\mathbf{j})
$$

or, in terms of autopower spectra:

$$
\mathbf{S}_{\mathbf{x x}}^{(\mathbf{j})}(\mathbf{i}, \mathbf{i})=|\mathbf{H}(\mathbf{i}, \mathbf{j})|^{2} \mathbf{S}_{\mathrm{ff}}(\mathbf{j}, \mathbf{j})
$$

where $\mathbf{S}_{\mathbf{x x}}^{(\mathbf{j})}(\mathbf{i}, \mathbf{i})$ is the autopower spectrum at target point $i$, due to path $j, \mathbf{S}_{\mathrm{ff}}(\mathbf{j}, \mathbf{j})$ is the autopower spectrum of the force measured at path $j$, and $\mathbf{H}(\mathbf{i}, \mathbf{j})$ is element $(i, j)$ of transfer function matrix $\mathbf{H}$.

For OTPA the procedure is as follows. From the definition of the transmissibility matrix $\mathbf{T}$, given by Eq. 3, the following expression can be derived to calculate the cross-spectral matrix $\mathbf{S}_{\mathbf{x x}}$ of the target responses from the cross-spectral matrix $\mathbf{S}_{\mathbf{y y}}$ of the indicator responses:

$$
\mathrm{S}_{\mathrm{xx}}=\mathrm{TS}_{\mathrm{yy}} \mathrm{T}^{\prime}
$$

The partial contributions of the vibrational sources near the indicator points $\mathbf{y}$ to the vibration level at the target points $\mathbf{x}$ can be determined by

$$
\mathbf{S}_{\mathbf{x x}}^{(\mathbf{j})}(\mathbf{i}, \mathbf{i})=|\mathbf{T}(\mathbf{i}, \mathbf{j})|^{2} \mathbf{S}_{\mathbf{y y}}(\mathbf{j}, \mathbf{j})
$$

where $\mathbf{S}_{\mathbf{y y}}(\mathbf{j}, \mathbf{j})$ is the autopower spectrum at response point $j$, and $\mathbf{T}(\mathbf{i}, \mathbf{j})$ is element $(i, j)$ of transmissibility matrix $\mathbf{T}$. Note the similarity of this expression with Eq. 14.

Before considering the partial contributions, we will first look at the response at the target points due to all transmission paths simultaneously. 


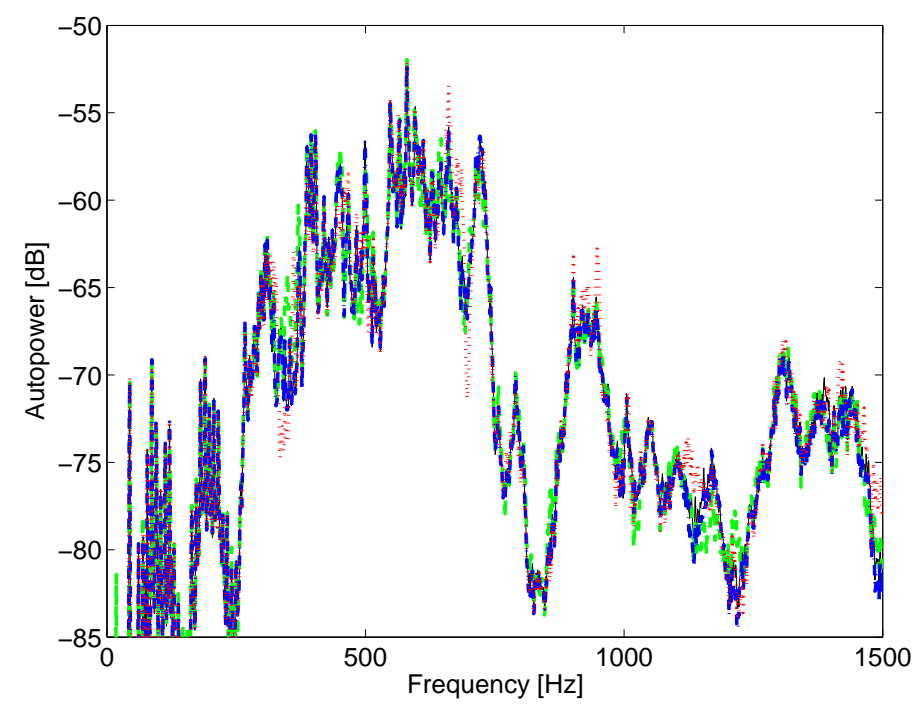

Figure 8: Reconstruction of the average level of the three target accelerations. Solid black line: measured data, dashed green line: using $\mathbf{H}$ and $\mathbf{S}_{\mathbf{f f}}$, dash-dotted blue line: using $\mathbf{T}_{\mathbf{o}}$ and $\mathbf{S}_{\mathbf{y y}}$, dotted red line: using $\mathbf{T}_{\mathbf{h}}$ and $\mathbf{S}_{\mathbf{y y}}$.

Though it is not a sufficient validation check, the reconstructions should obviously be able to give a good representation of the total response. Figure 8 shows the level of vibration of all three target accelerations, averaged in terms of the vibrational energy. Both the directly measured acceleration data and its reconstruction using four methods are shown, i.e. direct force measurement (employing Eq. 1, the measured forces and the measured transfer function to reconstruct the response), conventional OTPA and hammer excitation OTPA. It is clear that all reconstructions are able to predict the average level of the three target accelerations.

The partial contributions of each path, as predicted by the direct method that employs the measured forces, are drawn in Figure 9 together with the measured global response in terms of $1 / 24^{\text {th }}$ octave bands from $500 \mathrm{~Hz}$ till $1500 \mathrm{~Hz}$. It can be noted that at some frequencies below $750 \mathrm{~Hz}$, partial contributions can be greater than the global reconstruction, of up to $10 \mathrm{~dB}$ around $600 \mathrm{~Hz}$. This can be explained by a strong coupling between the active and passive parts of the system, caused by the eigenmodes of the structure.

The partial contributions of each path, as predicted by conventional OTPA are shown in Figure 10 in terms of $1 / 24^{\text {th }}$ octave bands from 500 $\mathrm{Hz}$ till $1500 \mathrm{~Hz}$. This figure shows that the conventional OTPA method fails 


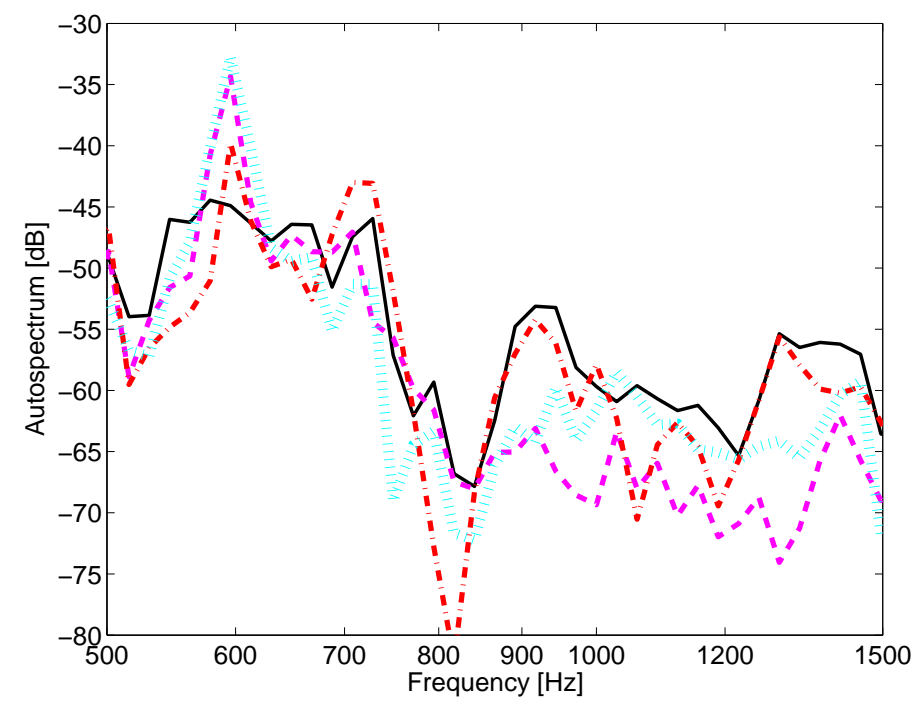

Figure 9: Reconstruction of the individual paths based on force measurements at interface, $1 / 24^{t h}$ octaves. Solid black line: Measured $\mathbf{S}_{\mathbf{x x}}$. Dashed purple line: Using $\mathbf{H}$ and $\mathbf{S}_{\mathrm{ff}}$, path 1. Dash-dotted red line: Using $\mathbf{H}$ and $\mathbf{S}_{\mathrm{ff}}$, path 2. Dotted magenta line: Using $\mathbf{H}$ and $\mathbf{S}_{\mathrm{ff}}$, path 3 .

to predict the significant peaks at $600 \mathrm{~Hz}$ for path 1 and path 3. However, this is not unexpected, as at such frequency there is a significant coupling between the transmission paths, which is difficult for an OTPA method to cope with [1]. At frequencies where there is only one path dominant, the OTPA method is partly able to predict the relevant transmission paths. In Figure 10 two of those frequency regions can be identified; at approximately $900 \mathrm{~Hz}$ and at approximately $1300 \mathrm{~Hz}$. But the quality of the estimate is not very good.

The partial contributions of each path, as predicted by hammer operational OTPA, again in terms of $1 / 24^{\text {th }}$ octave bands, are shown in Figure 11. Also here the method is unable to predict the strong interactions at $600 \mathrm{~Hz}$. At frequencies where there is only one path dominant, e.g. at approximately $900 \mathrm{~Hz}$ and at approximately $1300 \mathrm{~Hz}$, the quality of the estimation is better as compared to the conventional OTPA method. It is important to note that, even with a correctly estimated transmissibility matrix, the separation results are incorrect at most frequencies, with some exceptions at the mentioned frequencies. However, at frequencies where only one path is dominant, the prediction by means of OTPA (hammer or operational) is useful from an 


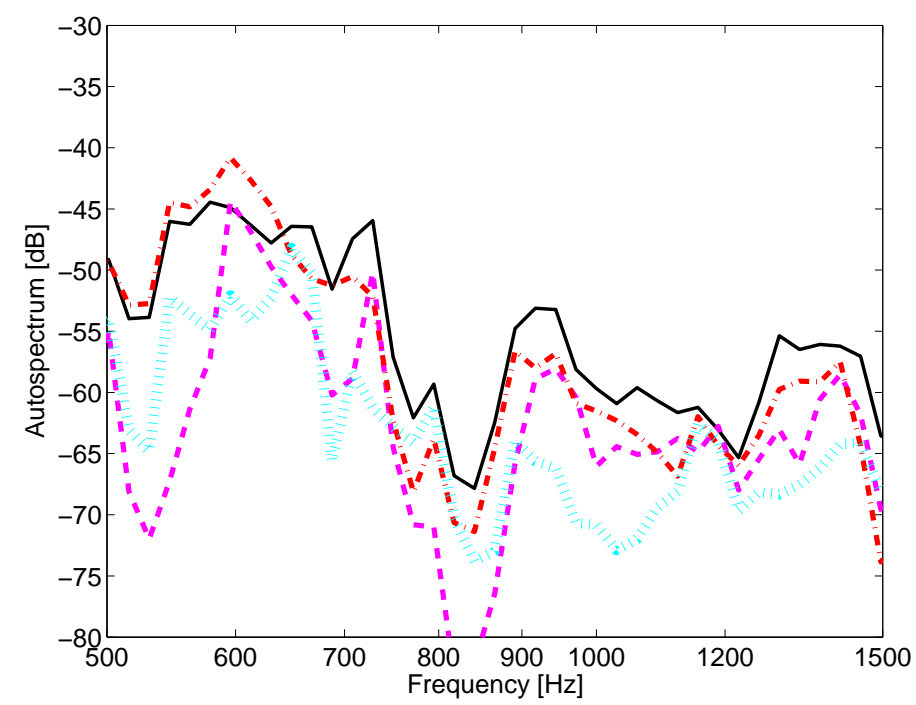

Figure 10: Reconstruction of the individual paths by means of conventional OTPA, $1 / 24^{\text {th }}$ octaves. Solid black line: Measured $\mathbf{S}_{\mathbf{x x}}$. Dashed purple line: Using $\mathbf{T}_{\mathbf{o}}$ and $\mathbf{S}_{\mathbf{y y}}$, path 1. Dash-dotted red line: Using $\mathbf{T}_{\mathbf{o}}$ and $\mathbf{S}_{\mathbf{y y}}$, path 2. Dotted magenta line: Using $\mathbf{T}_{\mathbf{o}}$ and $\mathbf{S}_{\mathbf{y y}}$, path 3 .

engineering point of view.

\section{Conclusion}

A measurement procedure was proposed to estimate the transmissibility matrix, using an artificial, external excitation. It was shown that this procedure gives more accurate estimates of the transmissibility matrix, as compared to the conventional one using machine operational vibrations (OTPA). The reason for this is that during external excitation, e.g. by means of an (non-instrumented) hammer, the structure is excited by hammer strokes which are largely independent from each other. In the conventional approach it is often difficult to obtain linearly independent operational deflection shapes even with strongly different operating conditions. This dependency generates difficulties during the matrix inversion, required to estimate the transmissibility matrix, causing inaccuracies in the transmissibility matrix estimates. The proposed measurement procedure does not have this problem,

Moreover, the experimental effort which is required is also less as compared to OTPA. The proposed method requires less artificial excitation ex- 


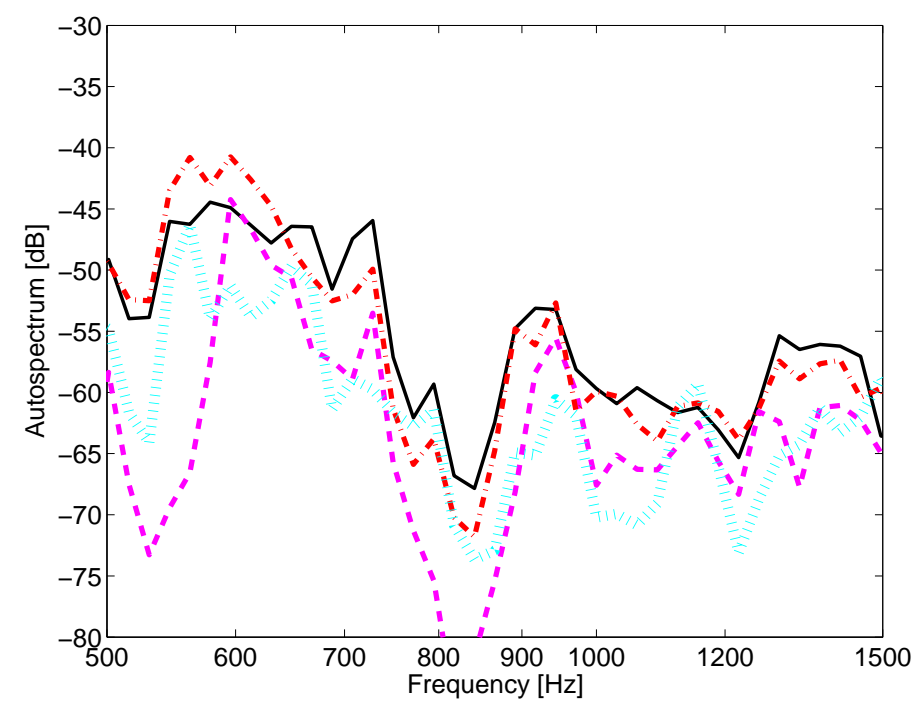

Figure 11: Reconstruction of the individual paths by means of hammer operational OTPA, $1 / 24^{t h}$ octaves. Solid black line: Measured $\mathbf{S}_{\mathbf{x x}}$. Dashed purple line: Using $\mathbf{T}_{\mathbf{h}}$ and $\mathbf{S}_{\mathbf{y y}}$, path 1. Dash-dotted red line: Using $\mathbf{T}_{\mathbf{h}}$ and $\mathbf{S}_{\mathbf{y y}}$, path 2. Dotted magenta line: Using $\mathbf{T}_{\mathbf{h}}$ and $\mathbf{S}_{\mathbf{y y}}$, path 3 .

periments, as compared to the number of operational conditions required for OTPA, again because of the fact that the responses of hammer strokes at well chosen locations are largely independent from each other.

As compared to traditional TPA the proposed method has the advantage in that it is not required to remove the vibration source which saves significant efforts.

In addition, a method is presented to identify the number of transfer paths during operation. The method is based upon a singular value decomposition of the response matrix. Using this approach, the number of most significant transfer paths can be estimated based on the normalized singular value distribution.

The proposed procedures are applied to a small gear-box test setup to estimate the number of significant transmission paths. Transmissibility matrices were estimated in a conventional way from operational machine vibrations and from hammer excitation vibrations. The conventional approach to estimate the transmissibility matrix typically took one to two hours, whilst the proposed procedure required only a few minutes, illustrating the reduced experimental efforts. 
For the specific test set-up at hand it was possible to estimate the transmissibility matrix rather precisely due to the inclusion of force transducers in the transmission paths, allowing to take the effect of pre-stress of the structure under the load of the gearbox into account. Using this transmissibility matrix as a reference, it was concluded that the errors in the transmissibility matrix estimates using hammer excitation were about one order of magnitude smaller than the transmissibility matrix estimates using operational machine vibrations, at relatively low experimental costs.

The application of OTPA to rank the individual transfer paths, however, were not very satisfying using either the conventional way or the proposed hammer excited transmissibility matrix estimate. It is important to note that, even with a correctly estimated transmissibility matrix, the ranking of the transmission paths can be incorrect. At frequencies where only one path is dominant, OTPA gives accurate results.

\section{Aknowledgement}

Bert Roozen, author of this paper, was an invited professor at INSA Lyon when this research was carried out. The people at INSA Lyon and especially Prof. Jean-Louis Guyader are gratefully acknowledged for giving me the opportunity to work with them.

\section{References}

[1] K. Janssens, P. Gajdatsy, H. Van der Auweraer, Operational Path Analysis: a critical review. In: Proceedings of ISMA Conference, 15-17 September, 2008, Leuven, Belgium.

[2] D. De Klerk, A. Ossipov, Operational transfer path analysis: Theory, guidelines and tire noise application. Mechanical Systems and Signal Processing 24 (2010) 1950-1962.

[3] C. Pezerat, J.L. Guyader, Identification of vibration sources, Applied Acoustics 61 (2000) 309-324

[4] J. Verheij, Multipath sound transfer from resiliently mounted shipboard machinery, PhD Dissertation, Technische Physische Dienst TNO-TH, Delft, 1986 
[5] Jan W. Verheij, Inverse and reciprocity methods for machinery noise source characterization and sound path quantification, part 1 and 2, International Journal of Acoustics and Vibration, Vol 2, No 1 and No 3, 1997

[6] M.H.A. Janssens, J.W. Verheij, A pseudo-forces methodology to be used in characterization of structure-borne sound sources, Applied Acoustics 61 (2000) 285-308.

[7] Q. Leclère, C. Pézerat, B. Laulagnet, L. Polac, Indirect measurement of main bearing loads in an operating diesel engine. Journal of Sound and Vibration 286 (2005) 341-361.

[8] H. Van der Auweraer, P. Mas, S. Dom, A. Vecchio, K. Janssens and P. Van de Ponseele, Transfer Path Analysis in the Critical Path of Vehicle Refinement: The Role of Fast, Hybrid and Operational Path Analysis In: Proceedings of the Noise and Vibration Conference and Exhibition, St. Charles, Illinois, May 15-17, 2007, SAE technical paper series 200701-2352.

[9] F.X. Magrans, Method of measuring transmission paths Journal of Sound and Vibration (1981) 74(3), 321-330.

[10] O. Guasch, F.X. Magrans, The Global Transfer Direct Transfer method applied to a finite simply supported elasticbeam Journal of Sound and Vibration 276 (2004) 335?359.

[11] O. Guasch, Direct transfer functions and path blocking in a discrete mechanical system, Journal of Sound and Vibration 321(2009) 854?874.

[12] Karl Janssens, Peter Gajdatsy, Ludo Gielen, Peter Mas, Laurent Britte, Wim Desmet, Herman VanderAuweraer, OPAX: A new transfer path analysis method based on parametric load models Mechanical Systems and Signal Processing 25(2011) 1321?1338.

[13] A.M.R. Ribeiro, J.M.M. Silva, N.M.M. Maia, On the generalisation of the transmissibility concept. Mechanical Systems and Signal Processing 14 (2000) 29-35. doi:10.1006/mssp.1999.1268

[14] D. Tcherniak, Application of the transmissibility matrix method to structure borne path contribution analysis. In: Proceedings of 
NAG/DAGA Conference, 23 - 26 March, 2009, Rotterdam, the Netherlands.

[15] P. Gajdatsy, K.Janssens, Wim Desmet, H.Van der Auweraer, Application of the transmissibility concept in transfer path analysis. Mechanical Systems and Signal Processing 24 (2010) 1963-1976.

[16] N.M.M. Maia, J.M.M. Silva, A.M.R. Ribeiro, The transmissibility concept in multidegree-of-freedom systems. Mechanical Systems and Signal Processing 15 (2001) 129-137.

[17] Q. Leclère, C. Pézerat, B. Laulagnet, L. Polac Application of multichannel spectralanalysis to identify the source of a noise amplitude modulation in a diesel engine operating at idle, Applied Acoustics, Volume 66, Issue 7, July 2005, Pages 779?798.

[18] S.M. Price, R.J. Bernhard, Virtual coherence: a digital signal processing technique for incoherent source identification. In: Proceedings of IMAC 4, 3-6 February, 1986, Los Angelos, California, USA.

[19] Okubo N. et Kumazawa K. Application of principal spectrum analysis to identify the vibration sources. In : Proceedings of ISMA 13, Leuven, Belgium, 1988

[20] M. Fontul, A.M.R. Ribeiro, J.M.M. Silva, N.M.M. Maia, Transmissibility matrix in harmonic and random processes. Shock and Vibration 11 (2004) 563-571.

[21] Q. Leclère, N.B. Roozen, C. Sandier, Experimental estimation of transmissibility matrices. In: Proceedings of ISMA-USD Conference, 17-19 September, 2012, Leuven, Belgium.

[22] D. Tcherniak, A.P.Schuhmacher, Application of Transmissibility Matrix Method to NVH Source Contribution Analysis. In: Proceedings of IMAC-XXVII, 9-12 February, 2009 Orlando, Florida, USA

[23] D. Otte, P. Sas, P. van de Ponseele, Principal component analysis for noise source identification. In: Proceedings of IMAC 6, 1-4 February, 1988, Kissimmee, Florida, USA. 